\title{
Phased-Array Design for Biological Clutter Rejection: Simulation and Experimental Validation
}

\author{
B. L. CHEONG \\ School of Meteorology, University of Oklahoma, Norman, Oklahoma
}

M. W. HOFFMAN

Department of Electrical Engineering, University of Nebraska, Lincoln, Nebraska

R. D. PALMER

School of Meteorology, University of Oklahoma, Norman, Oklahoma

STEPHEN J. Frasier AND F. J. LÓPEZ-DeKKeR

Department of Electrical and Computer Engineering, University of Massachusetts, Amherst, Massachusetts

(Manuscript received 23 March 2005, in final form 24 August 2005)

\begin{abstract}
This paper highlights recent results obtained with the Turbulent Eddy Profiler (TEP), which was developed by the University of Massachusetts. This unique $915-\mathrm{MHz}$ radar has up to 64 spatially separated receiving elements, each with an independent receiver. The calibrated raw data provided by this array could be processed using sophisticated imaging algorithms to resolve the horizontal structures within each range gate. After collecting all of the closely spaced horizontal slices, the TEP radar can produce threedimensional images of echo power, radial velocity, and spectral width. From the radial velocity measurements, it is possible to estimate the three-dimensional wind with high horizontal and vertical resolution. Given the flexibility of the TEP system, various array configurations are possible. In the present work exploitation of the flexibility of TEP is attempted to enhance the rejection of clutter from unwanted biological targets. From statistical studies, most biological clutter results from targets outside the main imaging field of view, that is, the sidelobes and grating lobes (if they exist) of the receiving beam. Because the TEP array's minimum receiver separation exceeds the spatial Nyquist sampling requirement, substantial possibilities for grating-lobe clutter exist and are observed in actual array data. When imaging over the transmit beam volume, the receiving array main lobe is scanned over a $\pm 12.5^{\circ}$ region. This scanning also sweeps the grating lobes over a wide angular region, virtually guaranteeing that a biological scatterer outside of the main beam will appear somewhere in the imaged volume. This paper focuses on suppressing pointlike targets in the grating-lobe regions. With a subtle change to the standard TEP array hardware configuration, it is shown via simulations and actual experimental observations (collected in June 2003) that adaptive beamforming methods can subsequently be used to significantly suppress the effects of point targets on the wind field estimates. These pointlike targets can be birds or planes with strong reflectivity. By pointlike the authors mean its appearance is a distinct point (up to the imaging resolution) in the images. The pointlike strong reflectivity signature exploits the capability of adaptive beamforming to suppress the interference using the new array configuration. It should be noted that this same array configuration does not exhibit this beneficial effect when standard Fourier beamforming is employed.
\end{abstract}

Corresponding author address: Boon Leng Cheong, School of Meteorology, University of Oklahoma, 100 East Boyd Street, Suite 1310, Norman, OK 73019.

E-mail: boonleng@ou.edu 


\section{Introduction}

Radar studies of the atmospheric boundary layer (ABL) have become widespread since the advent of relatively inexpensive and compact profiling radars (Ecklund et al. 1988), termed boundary layer radars (BLRs). Arguably, one of the most sophisticated of these types of radar systems is the so-called Turbulent Eddy Profiler (TEP), which was developed at the University of Massachusetts (Mead et al. 1998; Pollard et al. 2000). The TEP system is a volumetric radar designed for clear-air observations with high temporal and spatial resolution comparable to the grid size used in large eddy simulation (LES) models (Lilly 1967; Wyngaard et al. 1998). The multireceiver design of the TEP radar allows offline digital beamforming to construct volumetric images and is capable of acquiring measurements at altitudes up to $3 \mathrm{~km}$, depending upon atmospheric conditions. Imaging radars, including the TEP radar, help researchers and scientists to enhance their understanding of small-scale structure of the atmosphere. In addition to lower atmospheric measurements, imaging radars are also important for studies of other regions of the atmosphere, such as the mesosphere (Yu et al. 2001; Hysell et al. 2002), stratosphere (Rao et al. 1995), and ionosphere (Hysell 1996; Hysell and Woodman 1997).

For coherent radar imaging (CRI), signals from each of the receiver elements are combined to form a beam pointing in the direction of interest. The coherently combined signals allow spectral moments to be extracted from the beam-pointing direction. This technique is known as beamforming. By changing the beamforming weights, signals from arbitrary directions can be extracted. The beamforming weights can also be data adaptive. This allows suppression of strong signals outside the region of interest. With a high spatial resolution radar system, such as the TEP, many narrow beams can be formed over the field of view, providing tremendous detail in the imaging area. Pioneering work in spatial interferometry (SI), which is the predecessor to CRI, was accomplished in the early 1970s (Pfister 1971; Woodman 1971). Much work has been done to improve the angular resolution (Röttger and Vincent 1978) and since then, many atmospheric studies using SI have been conducted (e.g., Kudeki and Woodman 1990; Palmer et al. 1995). With the potential of CRI, many radar systems have been upgraded to include this function in order to conduct more detailed studies of the atmosphere. For example, the Middle and Upper Atmosphere (MU) radar (Fukao et al. 1985a,b) was upgraded to 25 receivers from 4 in order to utilize the capabilities of CRI. Newly developed commercial radar systems also feature multiple receivers allowing the use of CRI (Yu et al. 2001).

In addition to designing systems with a large number of receivers and a large aperture to improve the angular resolution (i.e., changing the hardware), alternate signal-processing algorithms can also be applied to improve the performance of CRI (i.e., changing the processing). By alternate signal-processing algorithms we mean that we are still employing CRI, but we are enhancing performance through data-dependent, or adaptive, beamforming. Such methods have been applied to atmospheric studies since the late 1990s (Palmer et al. 1998). One specific technique is inspired by a dataoptimized spectral estimation technique developed by Capon (1969) and is referred to as Capon beamforming, or Capon imaging. Statistical studies show that improved angular resolution is achieved using Capon beamforming as compared to traditional Fourier beamforming ( $\mathrm{Yu}$ et al. 2000). Given the large number of receivers that are available with the TEP radar, and the data-dependent beamforming techniques, the computational burden can be quite heavy. A computationally efficient implementation of these CRI beamforming algorithms for estimation of spectral moments that incurs no loss in CRI performance is termed pulse-pair beamforming (PPB) (Cheong et al. 2004b), and will be used in the present work. This algorithm is a computationally efficient combination of CRI and the pulse-pair processor, and it produces moment maps without the redundancy of synthesizing the beamforming output time series. It should be emphasized that PPB itself is not limited to any CRI technique. It can be fixed or data dependent and thus the terms Fourier PPB and Capon PPB will be seen throughout the rest of the paper.

One common and important problem for BLR, in general, is contamination from biological targets (e.g., birds and insects). Statistical studies have shown that most of this contamination occurs in the sidelobe and grating-lobe regions (Wilczak et al. 1995). The primary goal of the present work is to develop a method using imaging radars to suppress the interference from biological targets in the sidelobe and grating-lobe regions. By imaging radar we mean a combination of hardware that includes transmitter, multiple-antenna receivers, and software, which includes signal processing, imaging, and data analysis. Our primary goal of suppressing clutter interference is accomplished by implementing subtle changes in the array hardware configuration of the TEP radar system and employing data-dependent beamforming using the Capon PPB.

In this paper, the justification of the proposed array configuration is presented in section 2 . The section de- 
scribes the ad hoc search that was used to obtain the proposed design. In section 3, numerical simulation results are presented to compare the performance between the modified subarray and standard TEP configurations using both Fourier and Capon PPB. A point target with high reflectivity is used to simulate a bird flying across the main imaging region and eventually moving through a grating lobe. It should be emphasized that the clutter interference is assumed to have a pointtarget-like characteristic; that is, it has strong reflectivity and is point like. As such, this method is applicable to suppressing nonbiological point-target-like interference. A statistical analysis is conducted for data from both array configurations using the two PPB methods. In section 5, corroborating results from the field campaign conducted at the University of Massachusetts in June 2003 are presented.

\section{Motivation and merit of design}

The primary motivation of this work is to combat interference from biological targets that exist in the grating-lobe and sidelobe regions of the TEP system. Bird and insect echoes from these regions can angularly alias and appear in the imaging region. It is important to note that most birds are found in the grating lobes or sidelobes of the antenna (Wilczak et al. 1995). One solution to this problem is to design a transmit beam that only illuminates the main imaging area. Because of the inherent reflectivity of these nonatmospheric targets, however, even sidelobe echoes can be significantly stronger than atmospheric returns from the main lobe. Sidelobe echoes can be attenuated using adaptive beamforming methods. But, such techniques cannot eliminate biological clutter echoes from grating lobes. As a result, we will focus on the reduction of biological clutter strength that occurs in the grating lobes of the antenna array. Depending on the steering angle, a visible grating lobe may occur when a pair of receivers is separated by more than a one-half wavelength. For this situation there are angular locations where a signal from the desired direction and a distinct direction are both in phase at each of the receivers. In this case, phase steering alone cannot be used to separate the two signals. This angular position in the beam pattern where there is a replication in the gain is referred to as a grating lobe. The problem is particularly apparent in arrays that employ a regular lattice configuration for the receiver antennas. This is the case with the original hexagonal configuration of the TEP system with an antenna spacing of 1.73 times the wavelength. The grating lobes for a 61-element hexagonal TEP-like array are shown in Fig. 1a.
While these grating lobes occur for the regular hexagonal TEP configuration, one might imagine that the instance of an object actually moving through one of the lobes is relatively rare. However, the situation is more complicated because we use the radar to image (scan) a region of interest. As the main lobe is swept over a $12.5^{\circ}$ conical region, for example, the grating lobes are also swept over a larger region outside of the main transmit beam. Figures $1 \mathrm{~b}, 1 \mathrm{c}$, and $1 \mathrm{~d}$ show the locations of the grating lobes for the main-lobepointing directions of $\left(12.0^{\circ}, 0^{\circ}\right),\left(-7.5^{\circ},-7.5^{\circ}\right)$, and $\left(-8.0^{\circ},-9.0^{\circ}\right)$, respectively, for a TEP-like hexagonal configuration. The implication for radars using CRI is that a biological target flying outside of the main beam is quite likely to show up as a grating-lobe image aliased into the main imaging area for some location at some range gates. We see that the problem of grating-lobe clutter can be quite prevalent for imaging radars, especially at low altitudes where the level of biological activity is higher.

The signal-to-noise ratio (SNR) and radial velocity for a 2-h dataset are shown in Fig. 2. The data were acquired during the 2003 field campaign, which will be discussed in more detail in a later section. Quite often, biological targets fly horizontally and create parabolashaped echoes that can be clearly seen in the SNR data. The majority of the targets are situated within the sidelobe/grating-lobe regions because the main imaging region is limited to a $12.5^{\circ}$ azimuthal cone. With the flexibility of the TEP radar, various configurations are possible. As stated previously, our goal is reducing pointtarget-like interference through grating lobes. By slight modifications to the standard TEP array configuration, it will be shown in section 3 that adaptive beamforming methods can be applied to suppress the effects of the point-target-like interference (from a grating lobe) on both power and wind estimates.

To suppress correlated interference and reduce the effect of grating lobes, we investigated using multiple identical smaller hexagonal subarrays. A systematic search was used in order to find the optimal separation of the three subarrays. Figure 3 shows the new array configuration with three hexagonal subarrays and the array response of the system. The top-left panel shows the receiver positions of the entire array. The top-right panel shows the beam pattern of an array with the receivers being the center of each subsection. The bottom-left panel shows the beam pattern of an individual subarray. The bottom-right panel shows the composite beam pattern of the entire array, which is the product of the beam pattern in the top-right and bottom-left panels. By carefully adjusting the distance of each subarray 
(A) Beam at $\left(0.0^{\circ}, 0.0^{\circ}\right)$

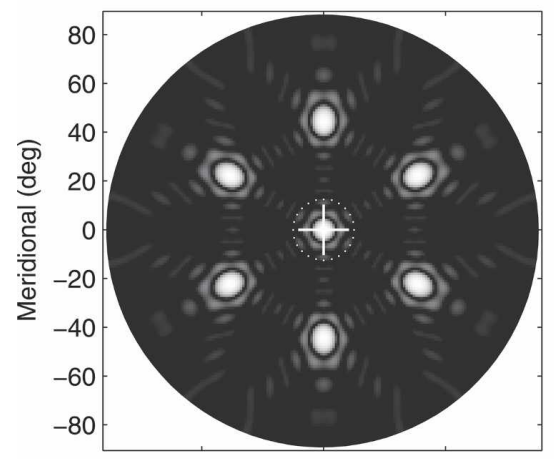

(C) Beam at $\left(-7.5^{\circ},-7.5^{\circ}\right)$

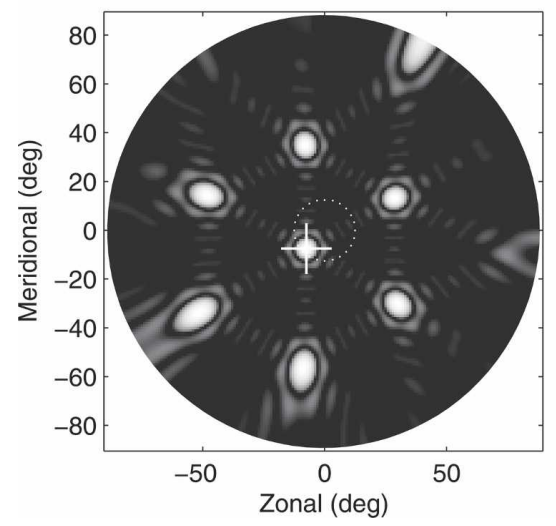

(B) Beam at $\left(12.0^{\circ}, 0.0^{\circ}\right)$
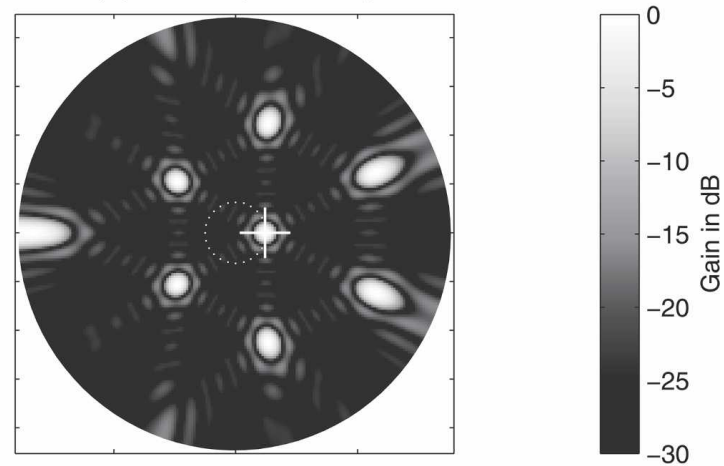

(D) Beam at $\left(-8.0^{\circ}, 9.0^{\circ}\right)$

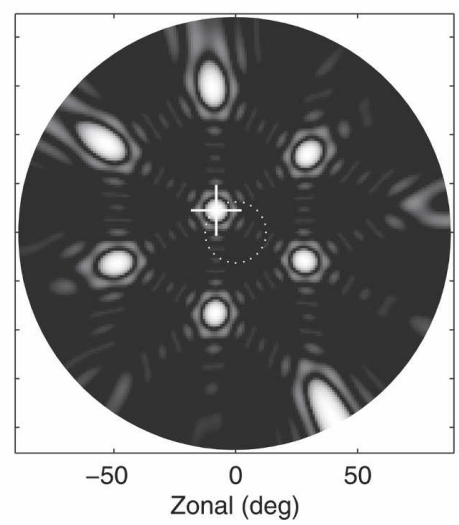

FIG. 1. Given the antenna spacing $d=0.566 \mathrm{~m}$, exceeding one-half of the wavelength of the 915-MHz TEP radar $(\lambda=0.328 \mathrm{~m})$, the resulting grating-lobe structures can be computed and are shown for a variety of main-beampointing angles.

from the center, one can place a null in the center of the grating-lobe regions of the subarray. Not only is the magnitude response in the grating-lobe region affected, but also the $2 \pi$ phase ambiguities are resolved in these regions (i.e., element pairs within a subarray maintain this ambiguity, while element pairs across subarrays do not). It would not have been possible to place nulls in these regions (via processing) for the total array response otherwise. Essentially, the original grating lobes have been converted into sidelobes with reduced gain compared to the main lobe and a distinctive three-lobe pattern. This three-lobe pattern dictates the structure of the aliased signal whenever a strong point target is present in the grating regions. It should be emphasized that one can now recognize pointlike targets that occur in the modified grating-lobe regions, rather than in the main lobe, via the three-blob structure in the image. It will be shown that by utilizing the adaptive Capon beamforming method that these sidelobes can be further suppressed, allowing significant attenuation of echoes from the original grating lobes. As a final note, when the main beam is steered throughout the $12.5^{\circ}$ conical transmit beam, the nulls are preserved in the center of the grating-lobe regions.

\section{Simulation study}

The numerical simulations presented here use the method of Cheong et al. (2004a), a modified version of Holdsworth and Reid (1995), with a simple reflectivity pattern shown in Fig. 4. Using radar imaging with the TEP radar, it is possible to reconstruct the reflectivity pattern as well as the wind field within the beam of the radar. In the context of this paper, the term "radar imaging" is limited in the horizontal domain only, that is, for a fixed range gate. Nonetheless, it is possible to produce closely spaced horizontal images to generate a three-dimensional volumetric image. The main goal of this simulated experiment is to investigate the bird clutter rejection issue; the model reflectivity is relatively unimportant. The point target used to simulate a bird is expected to appear as a sharp point (up to the resolution of the imaging system) and return strong radar echo because of its strong reflectivity. A uniform wind 

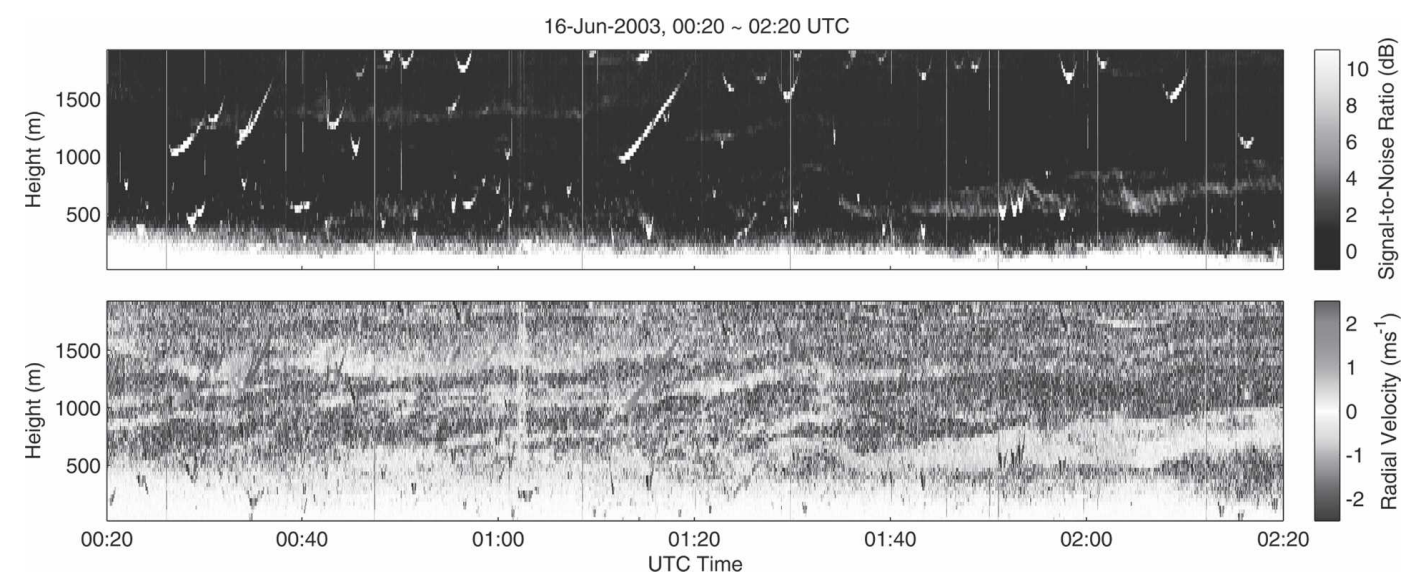

FIG. 2. Time history plots of zenith angle SNR and radial velocity for a 2-h dataset collected using the TEP radar and processed with a Fourier-based imaging method. Horizontally moving targets, such as birds, create parabolicshaped echoes and can be seen clearly in the image. Compared with the $12.5^{\circ}$ main imaging region, the sidelobe/ grating-lobe regions are much larger (cf. Fig. 1), and so it is suggested that most of these are the aliased signals created from the objects within the sidelobe/grating-lobe regions.

field is simulated with both the original hexagonal (61 element) and the proposed (57 element) subarray configurations.

Beam forming is used to image the reflectivity pattern and radial velocity map. For an $N$-receiver array, the beamforming process can be described mathematically as follows:

$$
y(n)=\mathbf{w}^{\dagger} \mathbf{x}(n),
$$

where $y(n)$ represents the output time series; $(\cdot)^{\dagger}$ represents the Hermitian operation; $\mathbf{x}(n)$ is an $N \times 1$ vector containing the receiving samples, usually direct current (dc) filtered to be zero mean; and $\mathbf{w}$ is an $N \times 1$ steering-weight vector. For traditional Fourier-based beamforming, the solution to the steering-weight vector is data independent. It is only a function of direction (zenith and azimuth angles) and is chosen to create constructive interference in the steering direction, described as follows:

$$
\begin{aligned}
\mathbf{w}_{\boldsymbol{f}} & =\frac{1}{\sqrt{n}}\left[e^{j \mathbf{k} \cdot \mathbf{D}_{1}} e^{j \mathbf{k} \cdot \mathbf{D}_{2}} \ldots e^{j \mathbf{k} \cdot \mathbf{D}_{N}}\right]^{\mathrm{T}}, \\
\mathbf{k} & =\frac{2 \pi}{\lambda}[\sin \theta \sin \phi \sin \theta \cos \phi \cos \theta],
\end{aligned}
$$

where $\theta$ and $\phi$ represent the zenith and azimuth angles, respectively, of the desired pointing direction. The three-dimensional position vector of receiver $i$ is represented by $\boldsymbol{D}_{i}$. The wavenumber vector and wavelength of the simulated radar system are denoted by $\mathbf{k}$ and $\lambda$, respectively. This weight vector is used in Eq. (1) if Fourier beamforming is considered.
A data-dependent-adaptive steering-weight vector can also be used in Eq. (1). A robust and well-known method was developed in the 1960s for seismic exploration (Capon 1969). The so-called Capon algorithm produces the steering-weight vector that minimizes the output power with a constraint to maintain unity gain at the beamforming direction. Thus, it minimizes the power leakage through the sidelobes. Let $\mathbf{w}_{c}$ denote the Capon-adaptive steering-weight vector. Detailed development of $\mathbf{w}_{c}$ can be found in the paper by Cheong et al. (2004b) and is summarized below,

$$
\mathbf{w}_{c}=\frac{\mathbf{R}_{\mathbf{x x}}^{-1}(0) \mathbf{e}}{\mathbf{e}^{\dagger} \mathbf{R}_{\mathbf{x x}}^{-1}(0) \mathbf{e}},
$$

where $\mathbf{R}_{\mathrm{xx}}(0)$ represents the zero-lag covariance matrix; $\mathbf{e}=\left[e^{j \mathbf{k} \cdot \mathbf{D}_{1}} e^{j \mathbf{k} \cdot \mathbf{D}_{2}} \ldots e^{j \mathbf{k} \cdot \mathbf{D}_{N}}\right]^{\mathrm{T}}$ is the steering vector for the desired look direction.

The CRI images of velocity and SNR presented here were produced using the PPB method described in (Cheong et al. 2004b). The method produces velocity and SNR images without the need to synthesize the actual time series. Using this procedure, significant computational savings are achieved. As the first step, an $N \times N$ covariance matrix is computed. Then, by using the definition of variance as the output power $P$ $=E\left[y(n) y^{\dagger}(n)\right]$ [assume $y(n)$ is zero mean], the returned power from a particular direction can be computed as follows:

$$
P(\theta, \phi)=\mathbf{w}^{\dagger} \mathbf{R}_{\mathbf{x x}}(0) \mathbf{w} .
$$


(A) Array Configuration

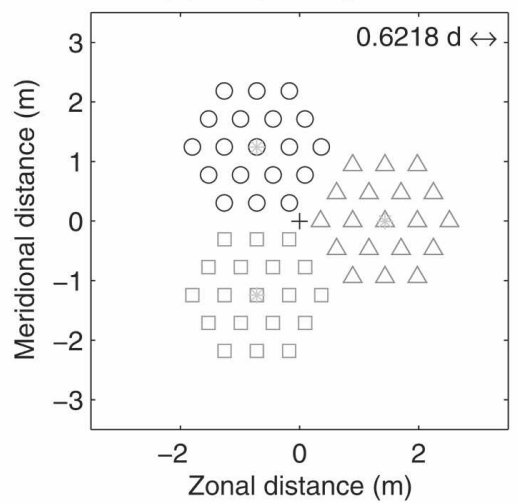

(C) Beampattern of Subarray

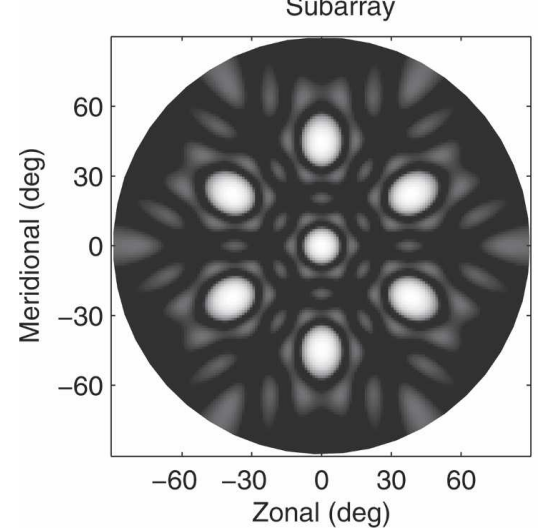

(B) Beampattern of 3-Receiver Array
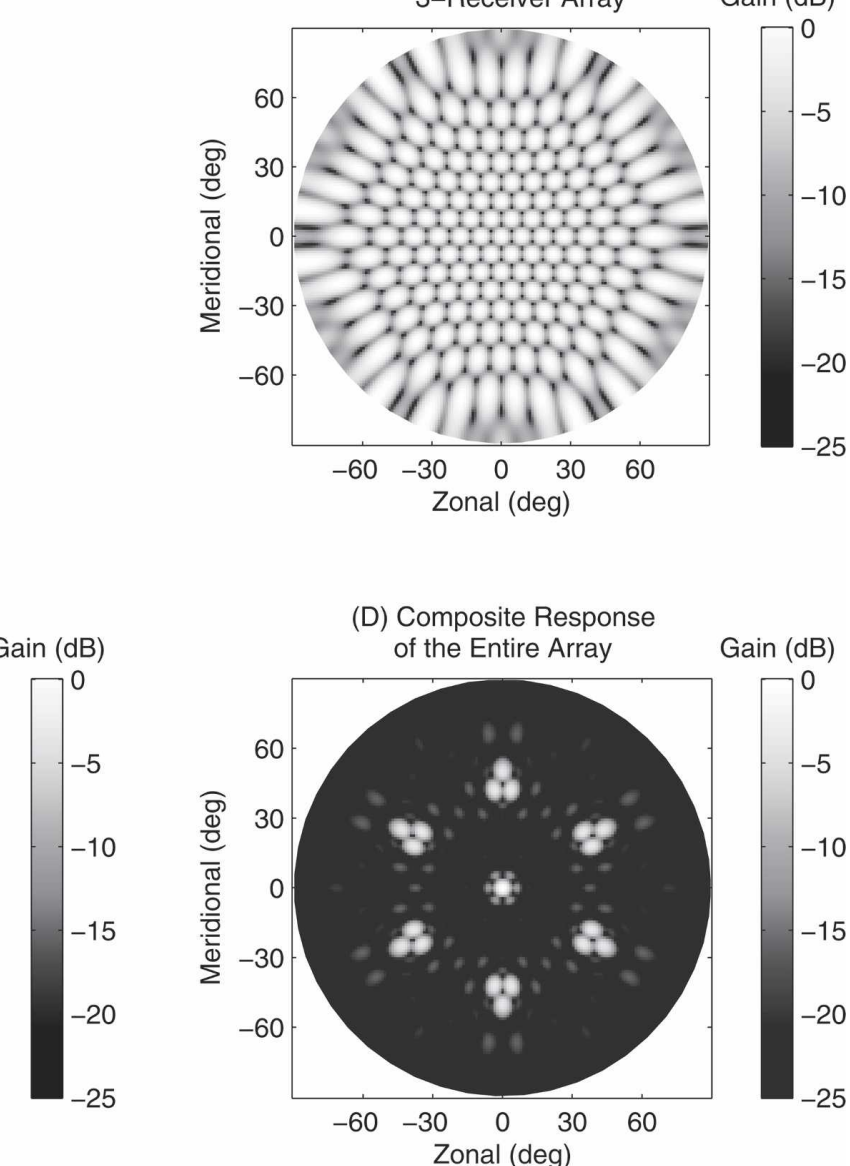

(D) Composite Response of the Entire Array

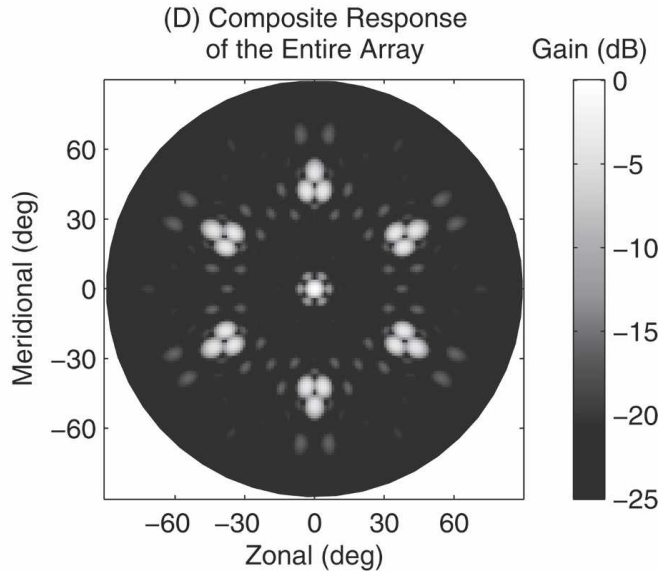

FIG. 3. (top left) The proposed TEP subarray configuration and (top right) the effective beam pattern of an array with three receivers being the center of each subarray are shown. We use the center of the array and the nearest receiver from each subarray to indicate the displacement spacing of each subarray. The optimum displacement was found to be 0.6218 times the standard antenna spacing. The noninteger factor indicates that the standard array baselines are broken, converting the grating lobes into three-lobe beam pattern. (bottom left) The three subarrays, which are designed to mimic the original TEP configuration and therefore inherit a similar beam pattern, are shown. Note that the displacements of the three subarrays are slightly different from the original element spacing. (bottom right) The composite beam pattern of the entire array is shown. One can see nulls in the center of each of the subarray grating lobes.

Radial velocity maps are obtained in a similar manner, which is described mathematically as

$$
v_{r}(\theta, \phi)=-\frac{\lambda}{4 \pi T_{s}} \arg \left[\mathbf{w}^{\dagger} \mathbf{R}_{\mathbf{x x}}\left(T_{s}\right) \mathbf{w}\right],
$$

where $\lambda=0.328 \mathrm{~m}$ is the wavelength of the transmit frequency of the TEP radar, and $T_{s}$ is the effective sampling time, that is, the interpulse period. We will refer to results obtained using $\mathbf{w}=\mathbf{w}_{f}$ as Fourier PPB and $\mathbf{w}=\mathbf{w}_{c}$ as Capon PPB throughout the rest of the paper. After estimating the radial velocity map, the three-dimensional wind field can be obtained using the standard Doppler beam-swinging (DBS) method adapted for the imaging radar case (Cheong et al. 2004b). Essentially, the three-dimensional wind field is estimated by the minimum least squares fit of the radial velocity field to the estimated "DBS" horizontal and vertical velocity.

One of the main goals of the TEP radar is to estimate the three-dimensional wind field with high angular resolution. Combined with Capon PPB, the proposed array configuration assists in achieving this goal by suppressing the effects of echoes from the grating-lobe regions in the wind field estimates. Figures 5 and 6 show the SNR images with superimposed horizontal wind fields from the Fourier PPB and Capon PPB, respec- 


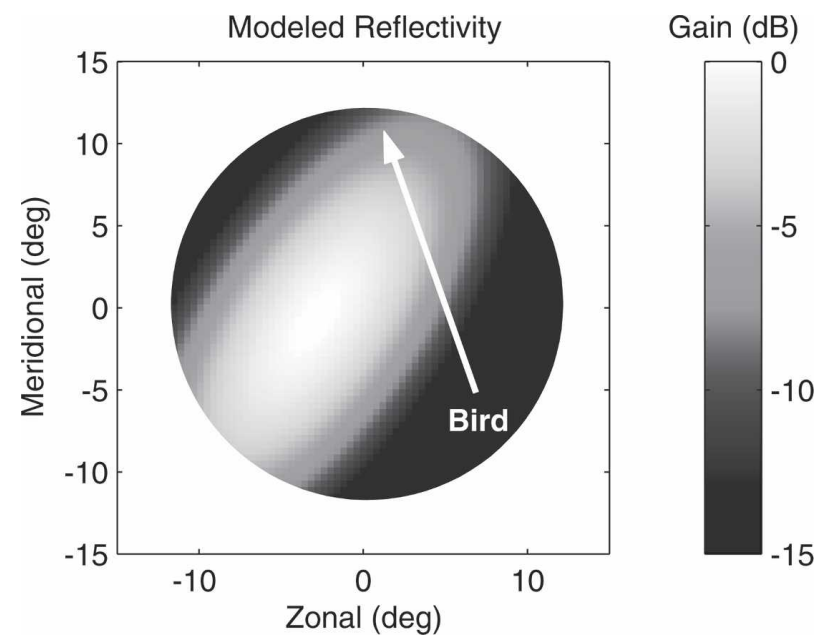

FIG. 4. Model reflectivity used in the simulation is a bivariate Gaussian function centered at $\left(-4^{\circ},-2^{\circ}\right)$. A significant bird target is placed at the southeast part of the imaged region at the beginning of the simulation. The bird is flying at a constant velocity toward the north-northwest.

tively. The top panels show the images obtained using the standard TEP configuration and the bottom panels show images obtained from the proposed subarray configuration. Note that the frame index is indicated in parentheses and the true horizontal wind reference arrow is shown in the upper-right corner.

Both Fig. 5 and Fig. 6 are obtained using the same simulation parameter for the bird. The bird moves through the main lobe of the antenna during the first few frames. In Fig. 5, the bird within the main lobe appears as a strong point target during frames 1-7. As the bird continues the fly, it eventually encounters a grating lobe and produces an aliased signal that appears in the imaging region in the standard TEP configuration. By using the proposed subarray configuration, the aliased signal appears as a three-blob echo in the images. These three-blob echoes result from the slight displacement of each subarray in the nonbaseline spacing (cf. Fig. 3). They no longer represent the standard grating lobe of the radar; the grating lobe is converted into sidelobes. Significant improvement can be achieved by using Capon PPB as the weights are adjusted to minimize the output image power at each pixel location, thus reducing the impact of sidelobe returns on the image.

Comparing Fig. 5 and Fig. 6, the Fourier processing is unable to eliminate the bird echo as the bird progresses through the grating-lobe regions. Although the bird is actually located at a comparably large angle, it is angularly aliased and appears close to zenith within the imaged region. As the echo progresses through the image, it has a distorting effect on the wind field estimates.
One of the reasons for the poor wind field estimates is the high-reflectivity variations within the images induced by the bird echoes (Cheong et al. 2004b). Finally, note that for the optimized array configuration, the Fourier results are different (but not significantly better) than the standard configuration results.

Considering the Capon PPB processing, in Fig. 6, the bird within the main lobe appears as a strong point target with both configurations of the array, similar to the previous case but with a better resolution. For the grating-lobe regions, however, the subarray configuration allows the suppression of the echoes from the grating-lobe regions. The standard TEP configuration does not because the grating lobes are indistinguishable from the main lobe. In the subarray configuration the threeblob echoes from the grating-lobe regions are not visible. The adaptive Capon PPB suppresses this strong interference from the bird by adjusting the weights to minimize the power leaking through the sidelobes near the grating-lobe regions. In addition, the horizontal wind field maps appear to be much improved compared to the other configuration/processing combinations. This improvement can be shown quantitatively.

Figure 7 illustrates the time history of the rms error of the wind field images of this simulation. During frames $1-7$, the bird appears as an ordinary strong reflectivity point target resulting in poor wind field estimates. The wind field estimate is poor in all cases regardless of the array configuration and the CRI method. When the bird flies through the grating-lobe region, however, the proposed array configuration combined with the Capon PPB method produces higher-quality wind field estimates than those from $\mathrm{Ca}$ pon PPB using the original TEP design, in which the aliased target can be seen because the grating lobes are indistinguishable from the main lobe. Given the fact that the majority of bird echoes occur from sidelobe/ grating-lobe echoes (Wilczak et al. 1995), the proposed array design warrants further study. To that end, an experiment was conducted using the proposed array configuration in June 2003. Results from that experiment will be presented in the next section.

\section{Experimental configuration}

Using the TEP radar facility, the proposed array configuration was implemented in Amherst, Massachusetts, in June 2003. Figure 8 shows the array configuration and a photo taken during the experiment. Shading on the left panel represents the measured height of the receiver elements. Although this will not be discussed here, the height information is important for proper calibration of the array. The trailer in the background 
TEP Configuration, Fourier Method, Average SNR $=3 \mathrm{~dB}$

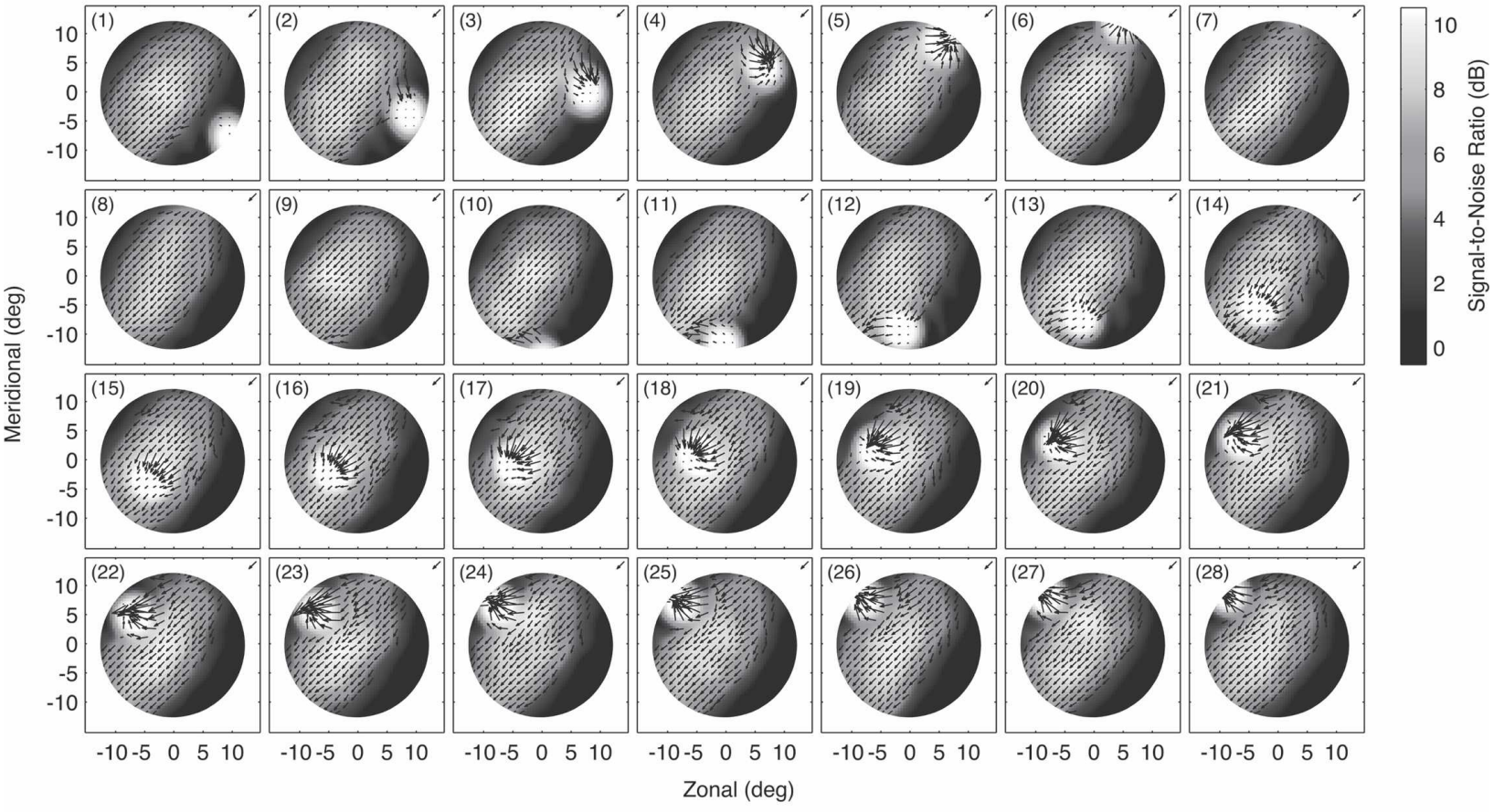

Subarray Configuration, Fourier Method, Average SNR $=3 \mathrm{~dB}$
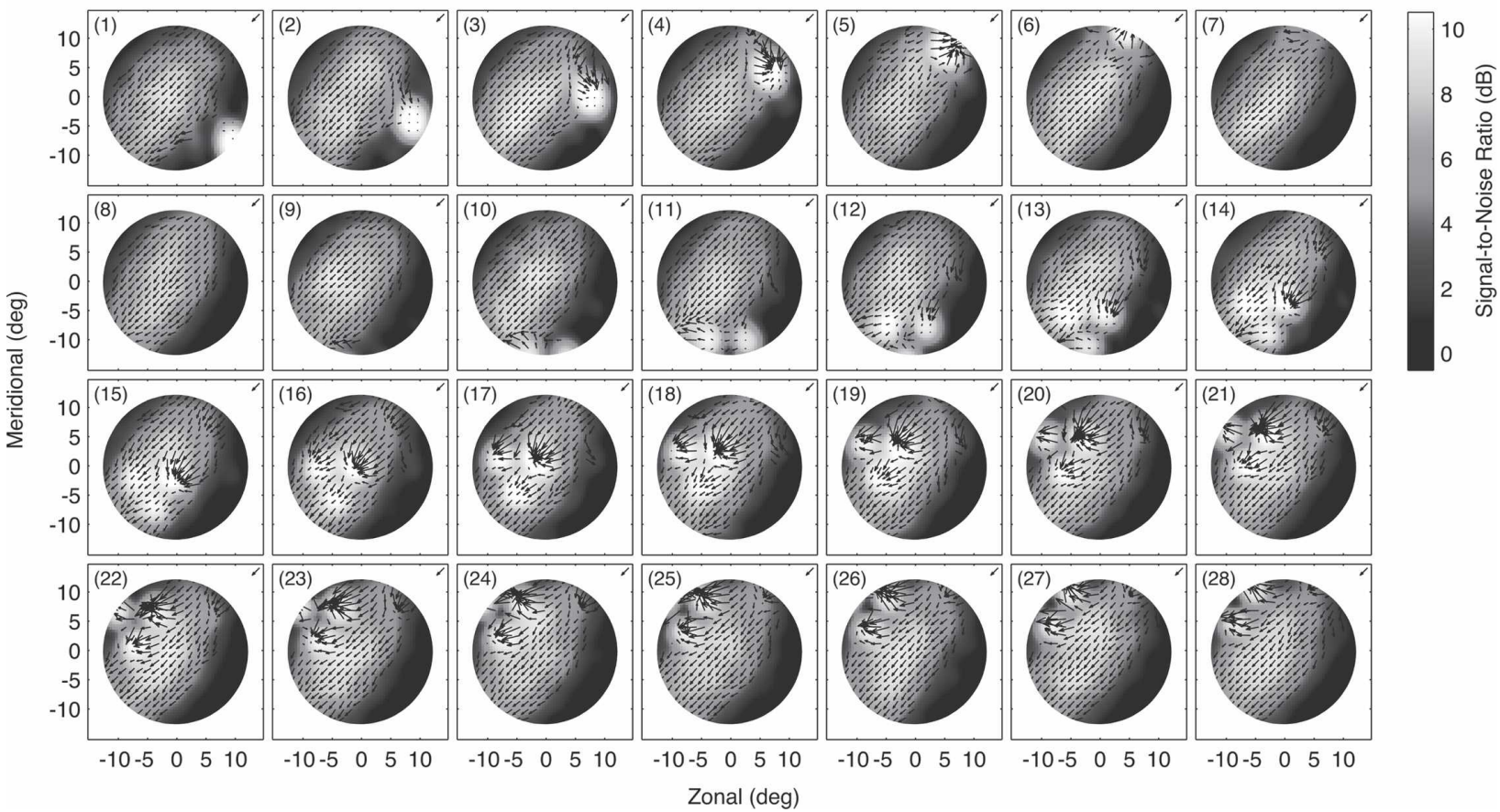

FIG. 5. Images obtained from Fourier PPB show a point target as expected when the bird is flying within the main imaging area (frames 1-7). As the bird continues to progress, it passes through a grating lobe and produces a point-target signal aliased in the standard TEP configuration. In the subarray configuration, however, the aliased signal appears as three-blob echoes in the background SNR image. Significant improvement can be obtained by using Capon PPB as shown in Fig. 6. Poor horizontal wind estimates are a consequence of the high variations of the reflectivity map (cf. Cheong et al. 2004b). 
TEP Configuration, Capon Method, Average SNR $=3 \mathrm{~dB}$

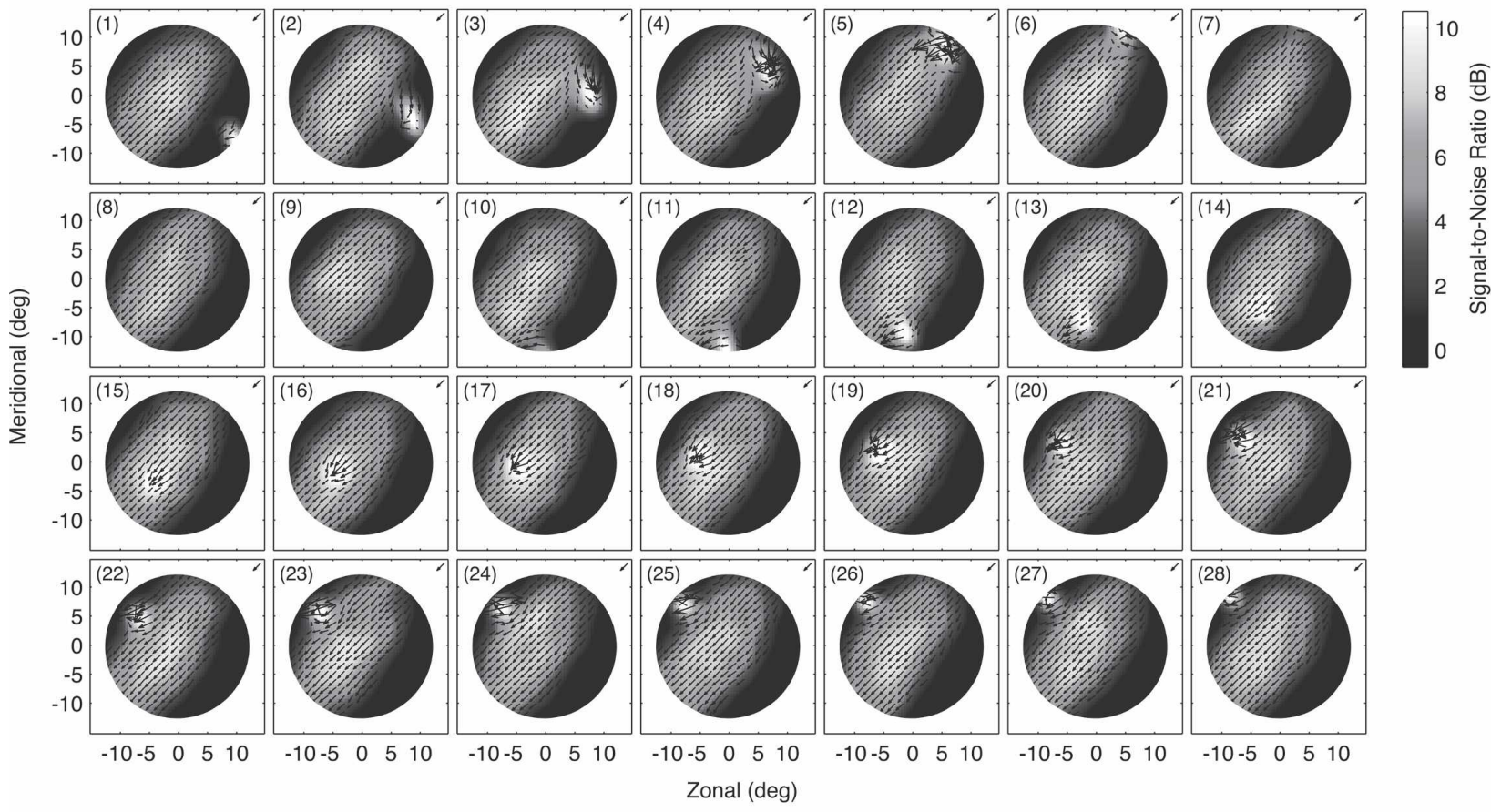

Subarray Configuration, Capon Method, Average SNR $=3 \mathrm{~dB}$
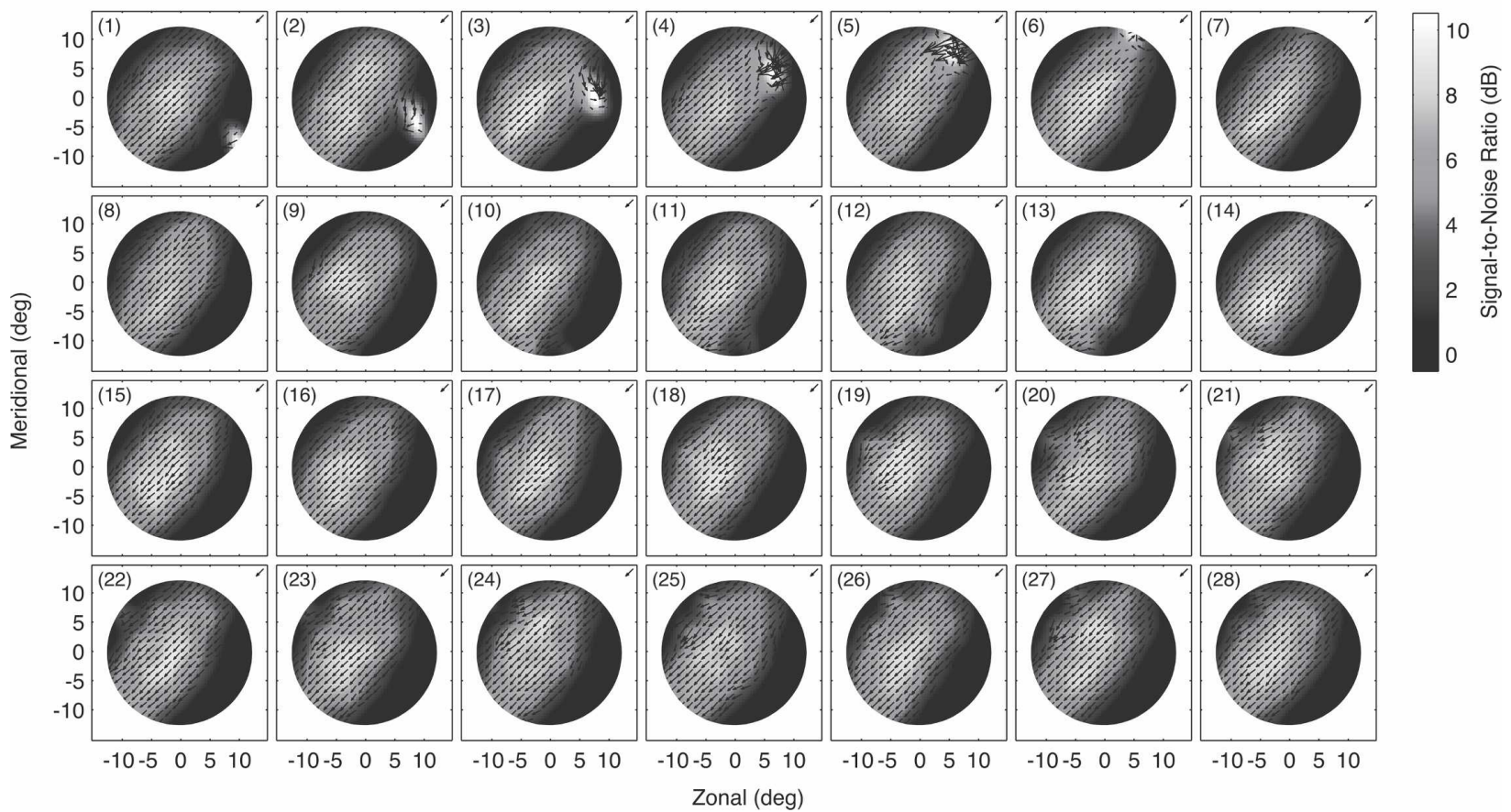

FIG. 6. Images obtained from Capon PPB show similar results with better resolution when the bird is within the main imaging area (frames 1-7). For the grating-lobe regions, however, the three-blob pattern is not observed. Capon PPB with the subarray configuration is capable of suppressing the returned echoes from the grating-lobe regions. This suppression is not as apparent when Capon PPB is used with the TEP configuration. Also, by reducing the aliased signal from the bird, the horizontal wind estimates are significantly improved. 


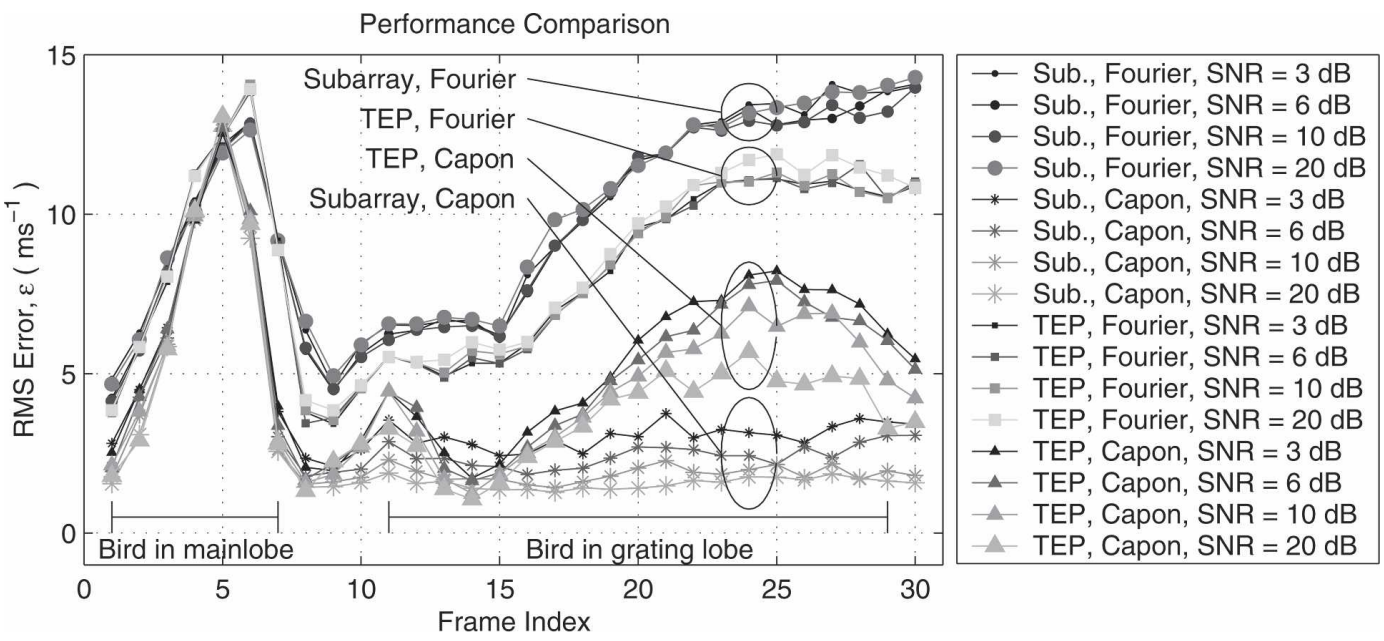

FIG. 7. This time history plot shows the rms error performance of wind estimates as the bird is flying through the main lobe and eventually through a grating lobe. During frames $1-7$, the birds are seen as an ordinary scatterer, regardless of the array configuration and the CRI method. When the bird is situated in the grating lobe during frames 11-28, however, the subarray configuration combined with Capon PPB provides significantly improved performance compared with the conventional array design.

of the photo houses the radar transmitter, receivers, and data acquisition system.

The $915-\mathrm{MHz}$ TEP radar antenna system is comprised of a transmit horn antenna and an array of microstrip antennas arranged in a hexagonal pattern. The transmitter's maximum power is $4 \mathrm{~kW}$, illuminating a $25^{\circ}$ conical volume through a vertically directed horn antenna. With a $25^{\circ}$ beamwidth transmit antenna, the radar covers approximately $440 \mathrm{~m}$ at an altitude of 1 $\mathrm{km}$. On the receiving side, an array of receiving anten- nas is placed on a flat platform to form a hexagonal lattice; the minimum separation antenna is approximately $0.57 \mathrm{~m}$ (Mead et al. 1998; Dekker and Frasier 2004). Each antenna has a $32^{\circ}$ receiving beamwidth and is connected to a low-noise amplifier and subsequently to the data acquisition system in the trailer. More detailed hardware information regarding the system can be found in Mead et al. (1998). The acquisition system uses a pulse-repetition frequency (PRF) of $35 \mathrm{kHz}$ with 250 coherent integrations resulting in an effective sam-
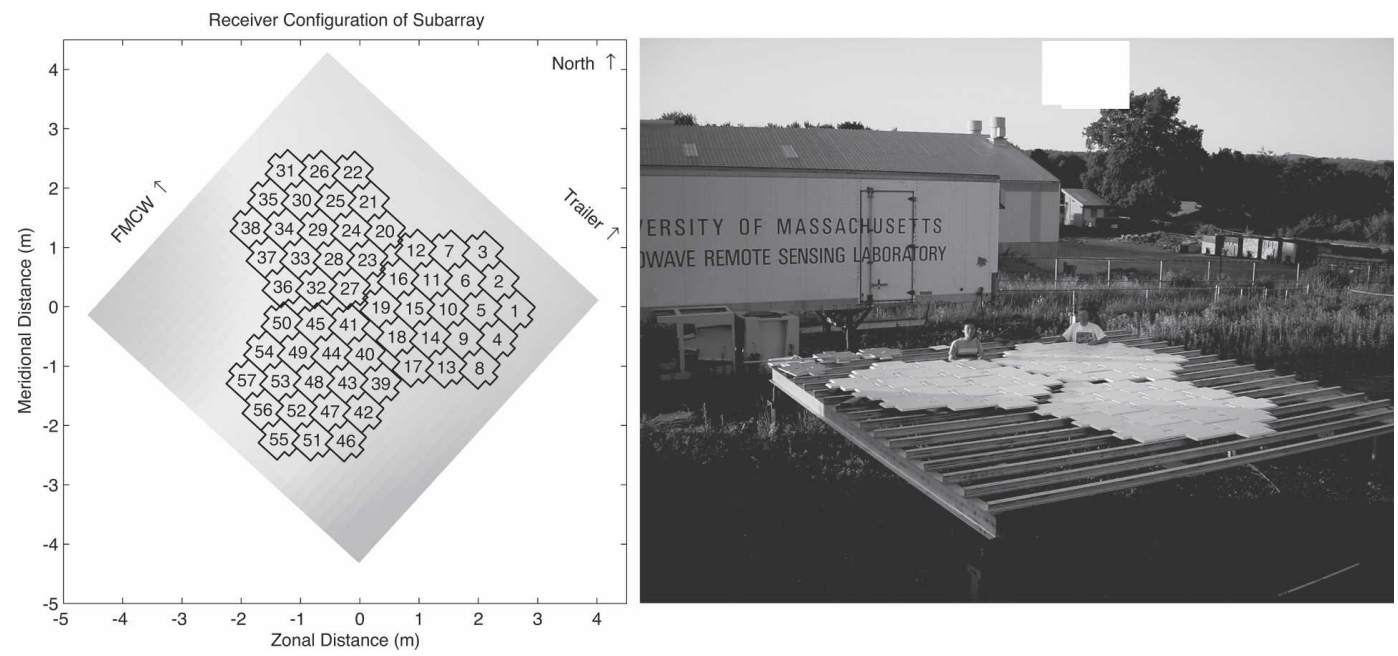

FIG. 8. (left) The array configuration on the platform is shown; the shading of the platform represents the height of the receiver elements. This information is crucial for accurate array calibration. (right) A picture taken during the campaign in 2003 is shown. 
pling rate of $7.14 \mathrm{~ms}$. The corresponding aliasing velocity at this given configuration is approximately 11.48 $\mathrm{m} \mathrm{s}^{-1}$. Because of the high sampling rate and large number of receivers, the system requires approximately $6 \mathrm{~GB}$ of storage space for each hour of data collection. The data are stored on disk for offline processing. A pulse width of $222 \mathrm{~ns}$ was used with a resulting 33.3-m range resolution. By using $\mathrm{PPB}$, signals from each receiver are coherently integrated in order to image the atmosphere within the illuminated region. The experimental results are presented in the next section.

\section{Results and discussion}

Example results from the experiment are presented in this section. Figure 2 in section 2 shows the conventional time history plot of the vertical beam SNR and radial velocity. The SNR plot shows the interference from birds and airplanes as parabolic shapes. Figure 9 shows an imaged view of the bird echo at approximately 0157 UTC at an altitude of approximately 1600 $\mathrm{m}$, with a temporal resolution of $7.5 \mathrm{~s}$. Images from Fourier and Capon PPB using the subarray configuration are shown in the top and the bottom panels, respectively. Each small image presents the SNR pattern for a particular range/time, arranged vertically for range and horizontally for time. The corresponding range for each row is indicated to the right of the figure, and the coordinated universal time stamp for each column is indicated on the top. Note that the imaging region is wider than the useful region $\left( \pm 12.5^{\circ}\right)$ in this example in order to more clearly demonstrate the clutter rejection capabilities using the proposed array configuration. The parabolic trend in range gates versus time can be observed in the series of images. By inspecting the spatial images in time, one can see that a bird is flying toward the northwest.

We were rather fortunate to have obtained an example that closely resembles the simulation example shown in section 3. At the beginning of the series of Fourier PPB images, the bird echo is aliased into the main imaging region as multiple three-blob echoes because of the natural beam pattern of the subarray configuration (cf. frame columns 2-7). During frame columns $8-13$, the bird is within the main imaging area and appears as a very strong signal. In fact, it saturates the chosen color scale compared with the results from $\mathrm{Ca}-$ pon PPB. Nonetheless, the strong point target can still be seen in the neighboring range gates. As the bird continues to fly, it eventually moves through a grating lobe (similar to our previous simulation) and appears as multiple three-blob echoes once more and moves in the same orientation. Note that the SNR level of the returned echoes of the main and grating lobes are comparable in the Fourier PPB method. However, images from the Capon PPB show that the aliasing problem is significantly suppressed as predicted from the numerical simulation. The suppression of the aliased signal consequently improves the power map generated from Capon PPB and thus produces higher-quality reflectivity maps. Because most biological targets are flying around the sidelobe/grating-lobe regions, this improvement definitely benefits reflectivity mapping and wind field estimates.

The entire 2-h set of data was processed using both the Fourier and Capon PPB methods and is presented in Fig. 10. These plots show the time history of the vertical pixel only (i.e., $\left.\left[0^{\circ}, 0^{\circ}\right]\right)$. Most of the interference shown in the plots is the result of leakage through the sidelobes, this also means that higher distortion may be found in another parts of the images (away from the vertical pixel, corresponding to grating lobes).

The top panel is exactly the same as that in Fig. 2. It is presented here for convenience for comparison to the results from Capon PPB method. In addition, the measurements from the Capon PPB are useful down to lower ranges than the ones from Fourier PPB. This provides a possibility of stable nocturnal boundary layer studies given that lower altitudes can be imaged reliably using the Capon PPB.

\section{Conclusions}

With an impetus to reduce biological clutter in an imaging radar, an approach to suppress interference from point-target-like echoes was presented in this paper. It is accomplished by using a new array configuration, termed subarray, combined with the data-adaptive Capon PPB method for signal processing. In the standard TEP configuration, targets from the main lobe and any grating lobe are indistinguishable simply because the inherent beam pattern shows identical gain for the main lobe and the grating lobes. A transmit antenna that has low power in the grating-lobe regions helps mitigate this problem, but the interference from physical objects such as birds and aircrafts is still visible and often saturates the images because of the inherent strong reflectivity. If the interference is in the main lobe, it cannot be eliminated, regardless of the beamforming techniques. However, it can be suppressed if it is in the grating-lobe regions. With a subtle change in the array configuration (hardware), the beam pattern of the array is also changed accordingly. The grating lobes are changed into three-blob sidelobes in the sub- 


\section{Fourier}

Signal-to-Noise Ratio ( dB )

\begin{tabular}{lll}
\hline & $\vdots$ \\
0 & 5 & 10
\end{tabular}

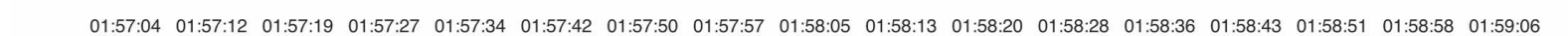
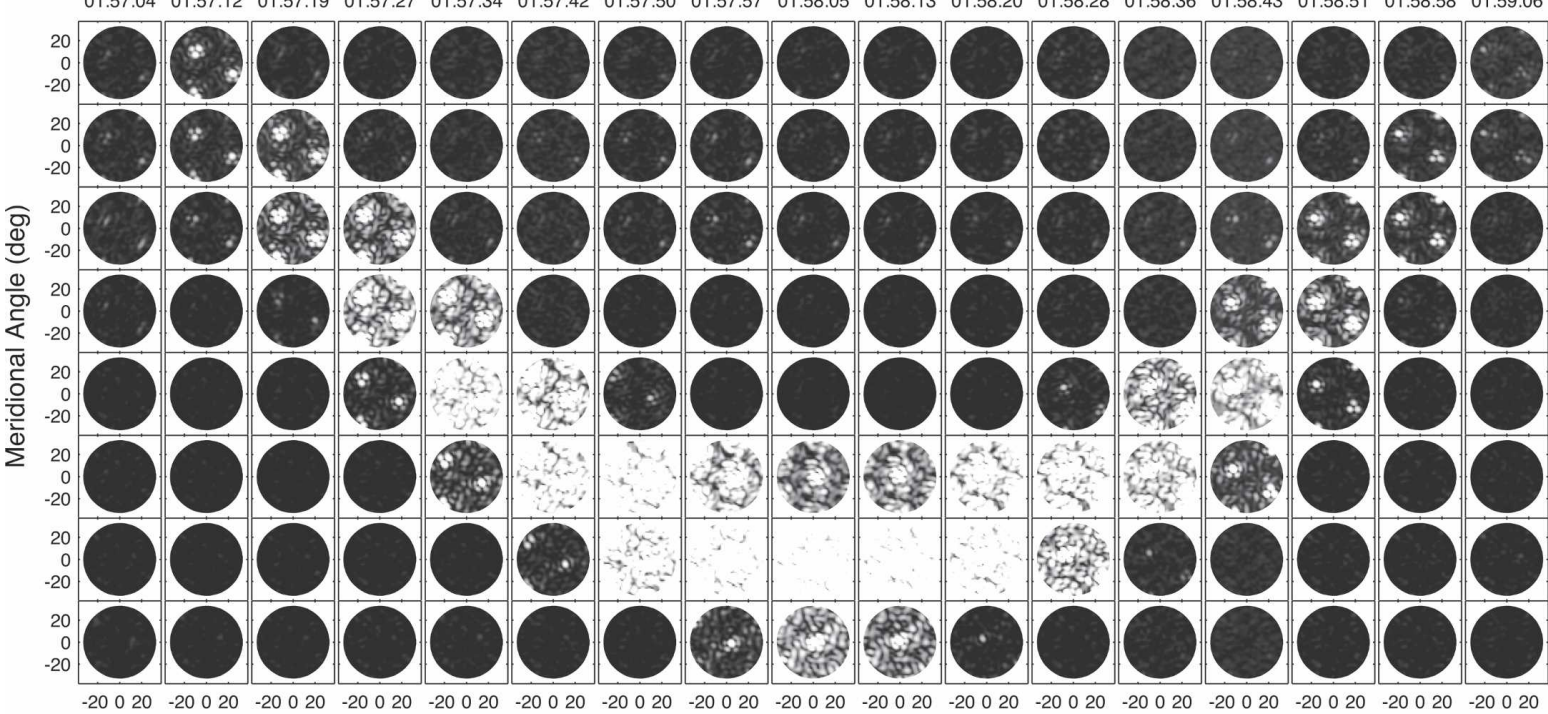

$1879 \mathrm{~m}$

Zonal Angle (deg)

\section{Capon}

Signal-to-Noise Ratio ( dB )

\section{0}

10

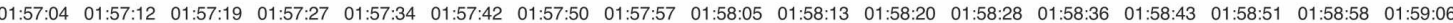

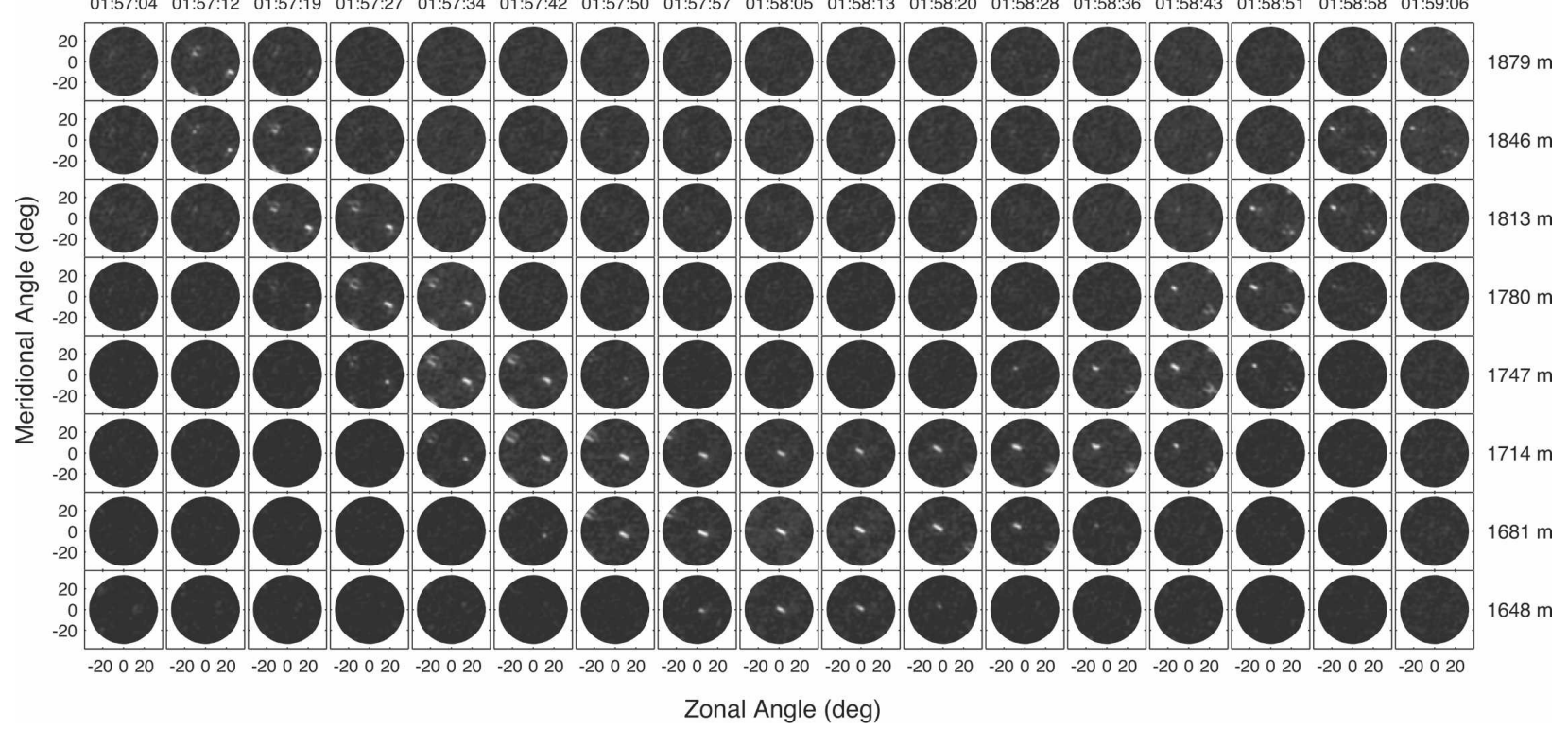

FIG. 9. Time range spatial history of reflectivity images from Fourier PPB and Capon PPB, range are displayed vertically with frame index 1-17 of each range displayed horizontally. A closer look at a bird flying toward northwest-west, around 0157 UTC, $1600 \mathrm{~m}$. Note that the imaging range in these images is wider than the useful region $\pm 12.5^{\circ}$, in order to more clearly demonstrate the effects of the clutter. The bird flies closer in range indicating that it is approaching the radar. When it is within the main lobe (frame columns 8-13), it appears as the dominant target in the series of images and power leakage through the sidelobes saturates the Fourier images. When it is in the grating lobes, however, the returned echoes are seen as three blobs in the Fourier PPB images. This signal has been greatly suppressed by using Capon PPB, as promised.

array configuration. Combined with the adaptive Capon PPB technique (signal processing), these sidelobes are further suppressed and, consequently, the aliased signals that are created from biological targets are re- duced. Aside from suppressing the echoes from biological targets in the grating lobes, we also observed that Capon PPB was able to image the atmosphere at very low altitudes (typically not obtained with Fourier PPB) 

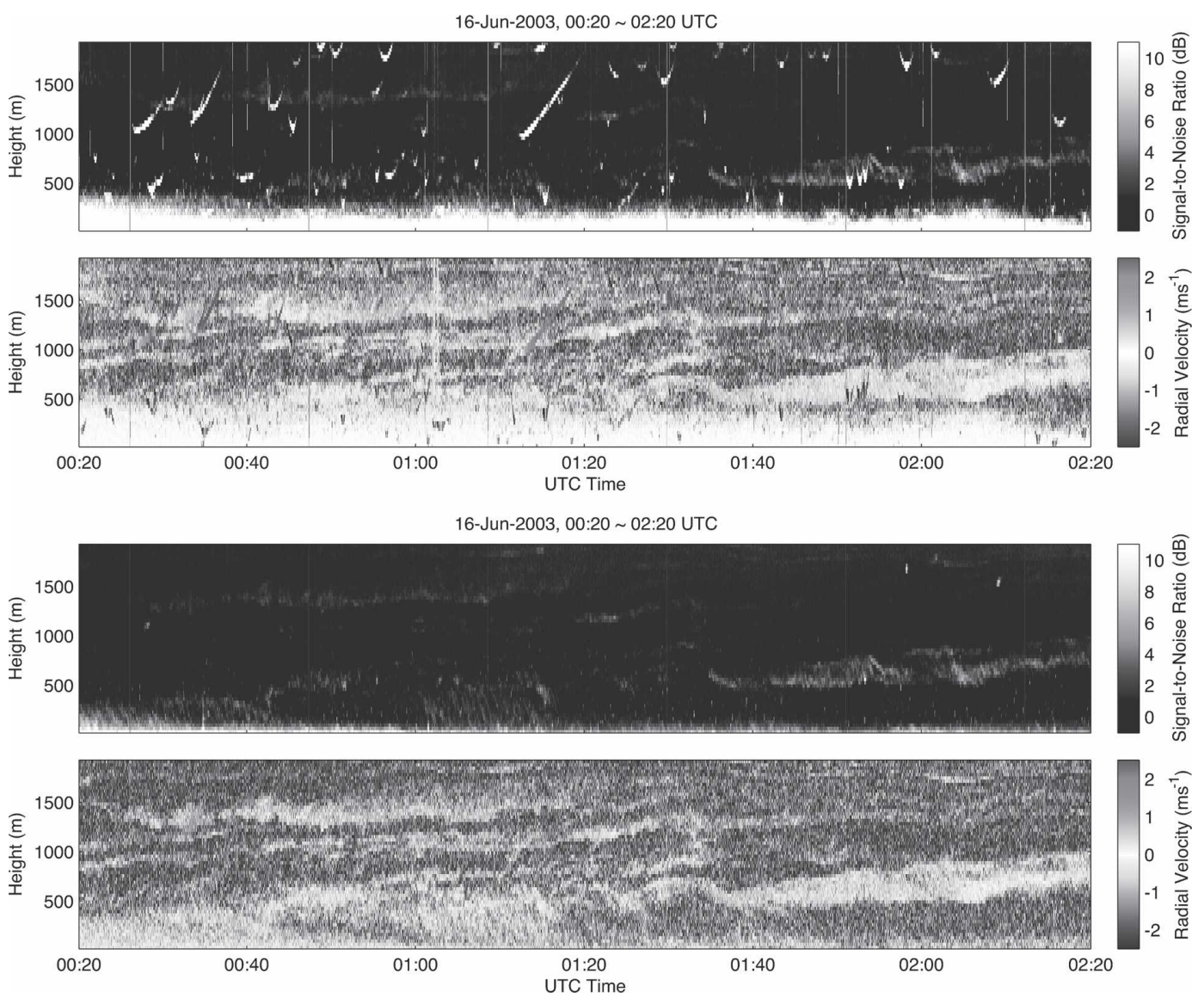

FIG. 10. Comparison of the time history plots generated from (top) Fourier and (bottom) Capon PPB. The top pair of panels is identical to Fig. 2 and is presented here for convenient reference, i.e., it is the time profile of the (top) zenith angle SNR and (bottom) radial velocity. The second pair of panels displays the same data processed with Capon PPB for the subarray data. The combination of subarray configuration and Capon PPB suppresses the interference from sidelobes/grating lobes. As can be seen, virtually all clutter is suppressed using the proposed subarray configuration combined with Capon PPB. The only instances when both images show the same interference are the ones in which targets are within the main lobe, e.g., 0027, 0157, and 0210 UTC, at or near the zenith angle.

and provides the possibility of studying the dynamics near the surface layer.

Acknowledgments. B. L. C., M. W. H., and R. D. P. were supported by the Army Research Office through Grant DDAD19-01-1-0407. S. J. F. and F. J. L.-D. were supported by the Army Research Office (Atmospheric Science) through Grant DDAAG55-98-1-0480. The authors thank V. Tellabati for his work on the calibration of the TEP array.

\section{REFERENCES}

Capon, J., 1969: High-resolution frequency-wavenumber spectrum analysis. Proc. IEEE, 57, 1408-1419.

Cheong, B. L., M. W. Hoffman, and R. D. Palmer, 2004a: Efficient atmospheric simulation for high-resolution radar imaging applications. J. Atmos. Oceanic Technol., 21, 374-378.
,,--- S. J. Frasier, and F. J. López-Dekker, 2004b: Pulse pair beamforming and the effects of reflectivity field variations on imaging radars. Radio Sci., 39, RS3014, doi:10.1029/2002RS002843.

Dekker, P. L., and S. J. Frasier, 2004: Radio acoustic sounding with a UHF volume imaging radar. J. Atmos. Oceanic Technol., 21, 766-776.

Ecklund, W. L., D. A. Carter, and B. B. Balsely, 1988: A UHF wind profiler for the boundary layer: Brief description and initial results. J. Atmos. Oceanic Technol., 5, 432-441.

Fukao, S., K. Wakasugi, T. Sato, S. Morimoto, T. Tsuda, I. Hirota, I. Kimura, and S. Kato, 1985a: Direct measurement of air and precipitation particle motion by very high frequency Doppler radar. Nature, 316, 712-714.

—, Y. Maekawa, T. Sato, and S. Kato, 1985b: Fine structure in mesospheric wind fluctuations observed by the Arecibo UHF Doppler radar. J. Geophys. Res., 90, 7547-7556.

Holdsworth, D. A., and I. M. Reid, 1995: A simple model of atmospheric radar backscatter: Description and application to 
the full correlation analysis of spaced antenna data. Radio Sci., 30, 1263-1280.

Hysell, D. L., 1996: Radar imaging of equatorial $F$ region irregularities with maximum entropy interferometry. Radio Sci., 31, 1567-1578.

— , and R. F. Woodman, 1997: Imaging coherent backscatter radar observations of topside equatorial spread $F$. Radio Sci., 32, 2309-2320.

— M. Yamamoto, and S. Fukao, 2002: Imaging radar observations and theory of type I and type II quasi-periodic echoes. J. Geophys. Res., 107, 1360, doi:10.1029/2002JA009292.

Kudeki, E., and R. Woodman, 1990: A poststatistics steering technique for MST radar applications. Radio Sci., 25, 591-594.

Lilly, D. K., 1967: The representation of small-scale turbulence in numerical simulation experiments. Proc. IBM Scientific Computing Symp. on Environmental Science, Yorktown Heights, NY, Thomas J. Watson Research Center, 195-210.

Mead, J. B., G. Hopcraft, S. J. Frasier, B. D. Pollard, C. D. Cherry, D. H. Schaubert, and R. E. McIntosh, 1998: A volume-imaging radar wind profiler for atmospheric boundary layer turbulence studies. J. Atmos. Oceanic Technol., 15, 849859.

Palmer, R. D., X. Huang, S. Fukao, M. Yamamoto, and T. Nakamura, 1995: High-resolution wind profiling using combined spatial and frequency domain interferometry. Radio Sci., 30, $1665-1679$.

, S. Gopalam, T. Yu, and S. Fukao, 1998: Coherent radar imaging using Capon's method. Radio Sci., 33, 1585-1598.
Pfister, W., 1971: The wave-like nature of inhomogeneities in the E-region. J. Atmos. Terr. Phys., 33, 999-1025.

Pollard, B. D., S. Khanna, S. J. Frasier, J. C. Wyngaard, D. W. Thomson, and R. E. McIntosh, 2000: Local structure of the convective boundary layer from a volume-imaging radar. $J$. Atmos. Sci., 57, 2281-2296.

Rao, P. B., A. R. Jain, P. Kishore, P. Balamuralidhar, S. H. Damle, and G. Viswanathan, 1995: Indian MST radar-Part I: System description and sample vector wind measurements in ST mode. Radio Sci., 30, 1125-1138.

Röttger, J., and R. A. Vincent, 1978: VHF radar studies of tropospheric velocities and irregularities using spaced antenna techniques. Geophys. Res. Lett., 5, 917-920.

Wilczak, J. M., and Coauthors, 1995: Contamination of wind profiler data by migrating birds: Characteristics of corrupted data and potential solutions. J. Atmos. Oceanic Technol., 12, 449-467.

Woodman, R. F., 1971: Inclination of the geomagnetic field measured by an incoherent scatter technique. J. Geophys. Res., 76, 178-184.

Wyngaard, J. C., L. J. Peltier, and S. Khanna, 1998: LES in the surface layer: Surface fluxes, scaling, and SGS modeling. $J$. Atmos. Sci., 55, 1733-1754.

Yu, T.-Y., R. D. Palmer, and D. L. Hysell, 2000: A simulation study of coherent radar imaging. Radio Sci., 35, 1129-1141.

,-- , and P. B. Chilson, 2001: An investigation of scattering mechanisms and dynamics in PMSE using coherent radar imaging. J. Atmos. Solar Terr. Phys., 63, 1797-1810. 